\title{
A HELLY NUMBER FOR UNIONS OF TWO BOXES IN R2
}

\author{
MARILYN BREEN \\ Department of Mathematics \\ University of Oklahoma \\ Norman, Oklahoma 73019 U.S.A. \\ (Received February 13, 1985)
}

ABSTRACT. Let $S$ be a polygonal region in the plane with edges parallel to the coordinate axes. If every 5 or fewer boundary points of $S$ can be partitioned into sets $A$ and $B$ so that conv $A U$ conv $B \subseteq S$, then $S$ is a union of two convex sets, each a rectangle. The number 5 is best possible.

Without suitable hypothesis on edges of $S$, the theorem fails. Moreover, an example reveals that there is no finite Helly number which characterizes arbitrary unions of two convex sets, even for polygonal regions in the plane.

KEY WORDS AND PHRASES. Helly - type theorems, unions of convex sets. 1980 MATHEMATICS SUBJECT CLASSIFICATION CODE. 52.A35, 52.A30, 52.A10.

1. INTRODUCTION.

We begin with some preliminary definitions. Let $S$ be a subset of $R^{d}$. $A$ point $x$ in $S$ is called a point of local convexity of $S$ if and only if there is some neighborhood $N$ of $x$ such that $S \cap N$ is convex. If $S$ fails to be locally convex at some point $q$ in $S$, then $q$ is called a point of local nonconvexity (1nc point) of $S$. For points $x$ and $y$ in $S$, we say $x$ sees $y$ via $S$ ( $x$ is visible from $y$ via $S$ ) if and only if the corresponding segment $[x, y]$ lies in $s$. Points $x_{1}, \ldots, x_{n}$ in $s$ are visually independent via $s$ if and only if for $1 \leqslant i<j \leqslant n, x_{i}$ does not see $x_{j}$ via $s$. Set $S$ is said to be 3-convex if and only if for every 3 points in $S$, at least one of the corresponding line segments lies in S. Finally, $S$ is called star-shaped if and only if there is some point $p$ in $S$ such that $p$ sees each point of $S$ via $S$. The set of all such points $p$ is the (convex) kernel of $\mathrm{S}$.

The following terminology will be used throughout the paper: Conv $\mathrm{S}$, bdry $\mathrm{S}$, and ker $\mathrm{S}$ will denote the convex hu11, boundary, and kernel of set $\mathrm{S}$, respectively, while lnc $S$ will be the set of 1 nc points of $S$. For distinct points $x$ and $y, R(x, y)$ will represent the ray from $x$ emanating through $y$. The reader is referred to Valentine [9] and to Lay [5] for a discussion of these concepts.

An interesting theorem by Lawrence, Hare, and Kenelly [4] provides the following characterization for unions of convex sets: For $S$ a subset of a linear 
topological space, $S$ is a union of $k$ convex sets if and only if each finite subset $F$ of $S$ has a k-partition $F_{1}, \ldots, F_{k}$ with conv $F_{i} \subseteq S, 1 \leqslant i \leqslant k$. A related problem concerns the existence of a finite Helly number to characterize such unions, and it is natural to ask when 'finite subset' in the hypothesis may be replaced by 'j-member subset' for an appropriate $j$. (See [3] for similar results.) Unfortunately, the Lawrence, Hare, Kenelly Theorem cannot be improved for $k=2$, even when $S$ is a compact subset of the plane. (See Example 3.) However, the problem of obtaining a finite Helly number to characterize certain unions of convex sets remains open. Recent results suggest sets for which such a theorem might hold. In work by Danzer and Grünbaum [2], a kind of Helly number (called a piercing number) is found for certain families of boxes. Moreover, in [1] another kind of Helly number (a Krasnosel'skii number for unions of starshaped sets) is established for certain polygonal regions whose edges are parallel to the coordinate axes. Therefore, it seems reasonable to examine such polygonal regions. This is the situation studied here, and the following result is obtained:

Let $S$ be a polygonal region in the plane with edges parallel to the coordinate axes. If every 5 or fewer boundary points of $S$ can be partitioned into sets $A$ and $B$ so that conv $A U \operatorname{conv} B \subseteq S$, then $S$ is a union of two convex sets, each a rectangle. The number 5 is best possible. Without suitable restrictions on the edges of $S$, the result fails and in fact no finite Helly number exists.

2. THE RESULTS.

The following lemma will be useful.

LEMMA 1. Let $S$ be a bounded subset of $R^{d}$, and let $k$ be a fixed integer, $k \geqslant 3$. If every $k$ or fewer boundary points of $S$ can be partitioned into sets $A$ and $B$ such that conv $A U$ conv $B \subseteq S$, then every $k$ or fewer points of $S$ can be partitioned in this way as well. Moreover, $S$ is 3 -convex.

PROOF. Clearly $S$ is closed. If $S$ is not connected, then it is easy to show that $S$ has exactly two components, each convex. Hence without loss of generality we may restrict our attention to the case in which $S$ is connected. Furthermore, we may assume that lnc $S \neq \emptyset$, for otherwise the closed, connected, locally convex set $S$ will be convex by a theorem of Tietze [6].

We begin the proof by showing that lnc $S \underline{c}$ ker $S$. Let $q \in \operatorname{lnc} S \neq \emptyset$. We assert that for every $z$ in bdry $S,[q, z] \subseteq S$ : Otherwise, since $S$ is closed, there would be a convex neighborhood $N$ of $q$ with no point of $N \cap S$ seeing $z$ via $S$. Since $q \in \operatorname{lnc} S, N \cap S$ is not convex, and standard arguments produce points $\mathrm{x}, \mathrm{y}$ in $\mathrm{N} \cap$ bdry $\mathrm{S}$ with $[\mathrm{x}, \mathrm{y}] \notin \mathrm{S}$. However, then points $\mathrm{x}, \mathrm{y}, \mathrm{z}$ would be visually independent points in bdry $\mathrm{S}$, contradicting our hypothesis. We conclude that $[q, z] \subseteq S$, and the assertion is established.

Now it is easy to show that $q \in$ ker $S$ : If not, then for some $w$ in $S$, $[q, w] \Phi s$, and $[q, w] \sim S$ would contain an open interval $(a, b)$ with $a, b \in$ bdry $\mathrm{S}$ and $\mathrm{q} \leqslant \mathrm{a}<\mathrm{b}<\mathrm{w}$. However, $[\mathrm{q}, \mathrm{b}] \subseteq \mathrm{S}$. Again we have a contradiction, and $q \in \operatorname{ker} s$, the desired result. 
Finally, to establish the lemma, let $x_{i} \in S, 1 \leqslant i \leqslant k$, and select $q \in$ lnc $S \neq \emptyset$. Without loss of generality, assume $q \notin\left\{x_{i}: 1 \leqslant i \leqslant k\right\}$, and let ray $R\left(q, x_{i}\right)$ meet bdry $S$ at $y_{i}$ with $q<x_{i} \leqslant y_{i}$. By our hypothesis, the points $\mathrm{y}_{1}, \ldots, \mathrm{y}_{\mathrm{k}}$ can be partitioned into sets $\mathrm{A}$ and $\mathrm{B}$ such that conv $\mathrm{A} U \operatorname{conv} B \subseteq S$. Relabeling if necessary, assume that $A=\left\{y_{1}, \ldots, y_{j}\right\}, \quad B=\left\{y_{j+1}, \ldots, y_{k}\right\}$. Since $q \in \operatorname{lnc} s, \operatorname{conv}(\{q\} \cup A) \subseteq S$ and $\operatorname{conv}\left\{x_{1}, \ldots, x_{j}\right\} \subseteq \operatorname{conv}(\{q\} \cup A) \subseteq S$. Similarly conv $\left\{x_{j+1}, \ldots, x_{k}\right\} \subseteq S$. Since $k \geqslant 3$, it is easy to see that $S$ is 3-convex, and the lemma is proved.

Without the requirement that set $S$ be bounded, Lemma 1 fails, as Example 1 illustrates.

EXAMPLE 1. Let $S$ be the set of points in $R^{2}$ which lie either in the first quadrant or on one of the coordinate axes. (See Figure 1.) The set bdry $S$ may be partitioned into sets $A$ and $B$ satisfying the hypothesis of the lemma. However, the conclusion of the lemma fails for every $k \geqslant 3$.

We are ready to establish the following Helly-type theorem for unions of rectangles in the plane.

THEOREM 1. Let $S$ be a polygonal region in the plane with edges parallel to the coordinate axes. If every 5 or fewer boundary points of $S$ can be partitioned into sets $A$ and $B$ so that conv $A U$ conv $B \subseteq S$, then $S$ is a union of two convex sets, each a rectangle. The number 5 is best posinhte.

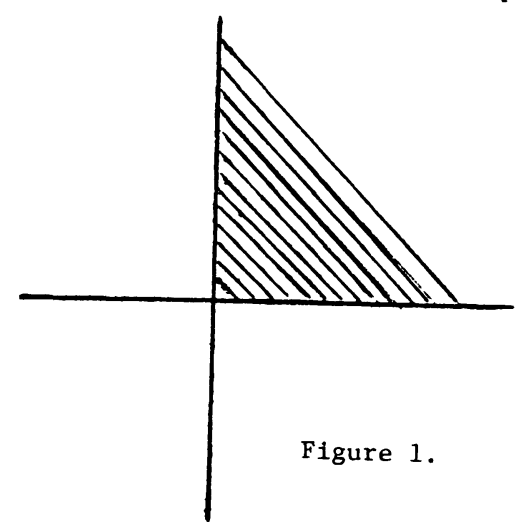

PROOF. We begin with some preliminary observations. By Lemma 1 , set $S$ is 3-convex. Moreover, using [8, Theorem 1], S is starshaped and lnc $\mathrm{S} \subseteq$ ker $\mathrm{S}$. Now in case $S$ has 0,1 , or 2 lnc points, we may use [8, Theorem 3] to conclude that $S$ is a union of two or fewer closed convex sets, the desired result. Therefore, throughout the argument it suffices to assume that $s$ has at least 3 inc points.

As in [7] and [1], it is helpful to order bdry $S$ in a clockwise direction. This in turn induces a natural order on the edges of $\mathrm{S}$, and we may classify each edge as 'right', 'left', 'up', or 'down' according to the order it inherits from bdry S. For convenience of notation, we label the lnc points of $S$ by $q_{1}, q_{2}, \ldots, q_{n}$, again according to the clockwise order on bdry $s$. Recall that $n \geqslant 3$ by a previous assumption. The proof of the theorem will require some results concerning these Inc points. 
It is easy to see that for each 1 nc point of $S$, exactly one of the following must occur relative to our order: The lnc point is either preceded by a 'right' edge and followed by an 'up' edge, preceded by a 'left' edge and followed by a 'down' edge, preceded by a 'down' edge and followed by a 'right' edge, or preceded by an 'up' edge and followed by a 'left' edge. Moreover, using this classification together with the 3-convexity of $\mathrm{s}$, it is not hard to show that no three

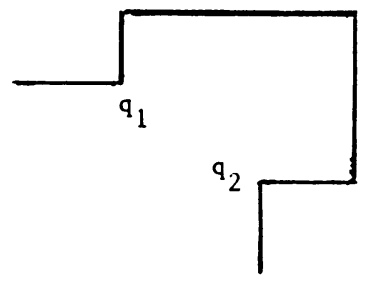

Case 1 .

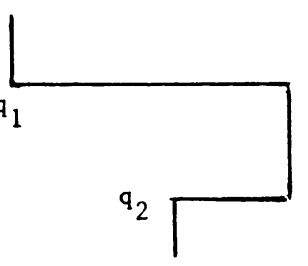

Case 4 .

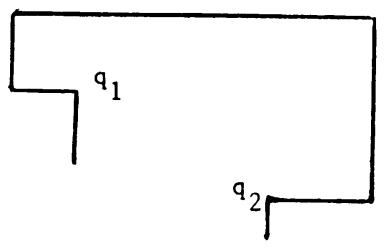

Case 7 .

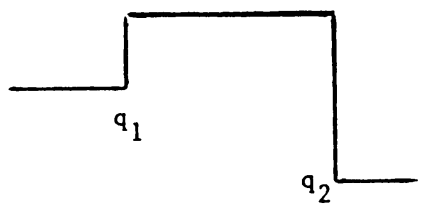

Case 2.

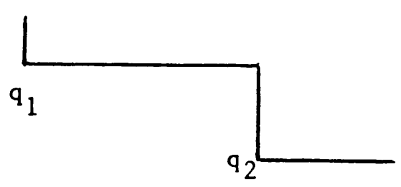

Case 5 .

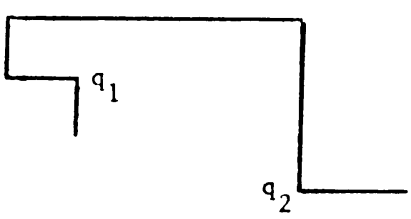

Case 8 .

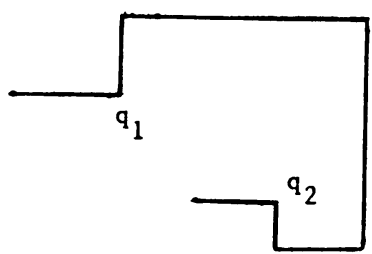

Case 3 .

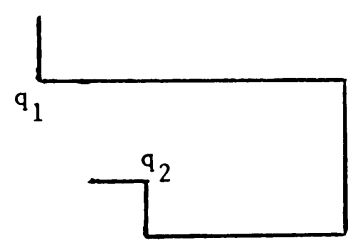

Case 6.

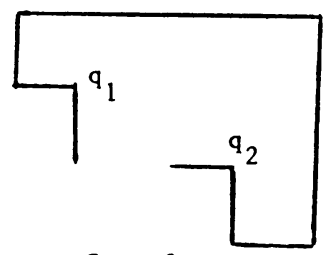

Case 9.

Figure 2.

consecutive lnc points of $S$ (in our order) can be collinear: Otherwise, for an appropriate orientation of $\mathrm{S}$, this would yield a 'right' edge whose predecessor is a 'down' edge and whose successor is an 'up' edge, producing three visually independent points of $\mathrm{S}$, impossible.

Furthermore, we assert that every two consecutive lnc points of $S$ determine a segment parallel to one of the coordinate axes: Suppose on the contrary that the condition fails for $q_{1}$ and $q_{2}$. Without loss of generality, assume that $s$ is oriented in the plane so that $q_{1}$ is to the left of $q_{2}$ and above $q_{2}$. We examine possible classifications for $q_{1}$ and $q_{2}$. Using the fact that $q_{2}$ follows $q_{1}$ in our clockwise order, it is not hard to see that $q_{1}$ cannot be preceded by a 'left' edge and followed by a 'down' edge. For convenience of terminology, we say that $q_{1}$ cannot be classified as 'left-down'. Similarly, $q_{2}$ cannot be classified as 'right-up'. 
Examine the remaining 9 possibilities. (See Figure 2, Cases 1-9, for corresponding illustrations.)

1. $\mathrm{q}_{1}$ is 'right-up' and $\mathrm{q}_{2}$ is 'left-down'

2. $\mathrm{q}_{1}$ is 'right-up' and $\mathrm{q}_{2}$ is 'down-right'

3. $\mathrm{q}_{1}$ is 'right-up' and $\mathrm{q}_{2}$ is 'up-left'

4. $\mathrm{q}_{1}$ is 'down-right' and $\mathrm{q}_{2}$ is 'left-down'

5. $\mathrm{q}_{1}$ is 'down-right' and $\mathrm{q}_{2}$ is 'down-right'

6. $\mathrm{q}_{1}$ is 'down-right' and $\mathrm{q}_{2}$ is 'up-left'

7. $\mathrm{q}_{1}$ is 'up-left' and $\mathrm{q}_{2}$ is 'left-down'

8. $\mathrm{q}_{1}$ is 'up-left' and $\mathrm{q}_{2}$ is 'down-right'

9. $\mathrm{q}_{1}$ is 'up-left' and $\mathrm{q}_{2}$ is 'up-left'

It is not hard to prove that each of Cases 2 through 9 above violates the condition that $q_{1}, q_{2} \in$ ker $s$, so none of these can occur. Thus Case 1 is the only possibility.

Moreover, by a similar argument involving the classification of $1 \mathrm{nc}$ point $\mathrm{q}_{3}$, $\mathrm{q}_{3}$ must be 'up-left': Classification 'down-right' is impossible in our clockwise order. Classifications 1)'right-up' and 2)'left-down' violate the facts that $\mathrm{q}_{1} \in \operatorname{ker} \mathrm{S}$ and $\mathrm{q}_{2} \in \operatorname{ker} \mathrm{S}$, respectively. (See Figure 3, Cases 1-2.) However, this forces $S$ to have 5 boundary points $p_{i}, 1 \leqslant 1 \leqslant 5$, for which there is no partition satisfying our hypothesis: Select $p_{1}$ near $q_{1}$ on the edge preceding $\mathrm{q}_{1}, \mathrm{p}_{2}$ above $\mathrm{q}_{1}$ and right of $\mathrm{q}_{2}, \mathrm{p}_{3}$ near $\mathrm{q}_{2}$ on the edge

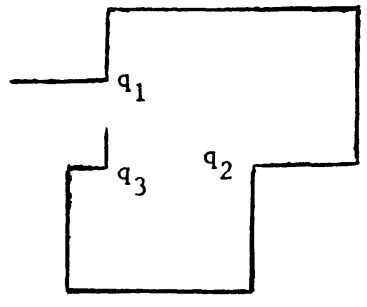

Case 1.

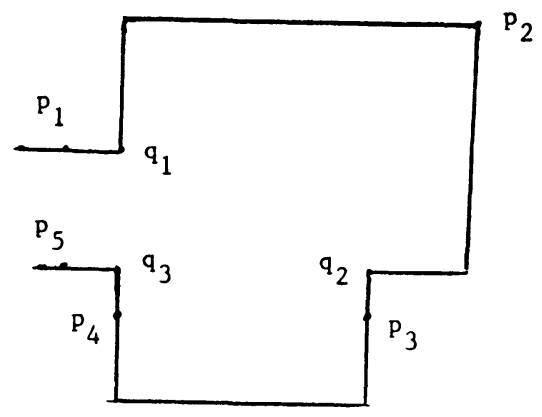

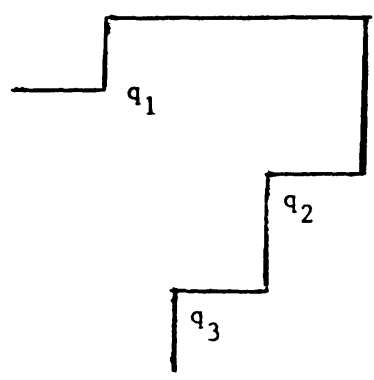

Case 2.

Figure 3.

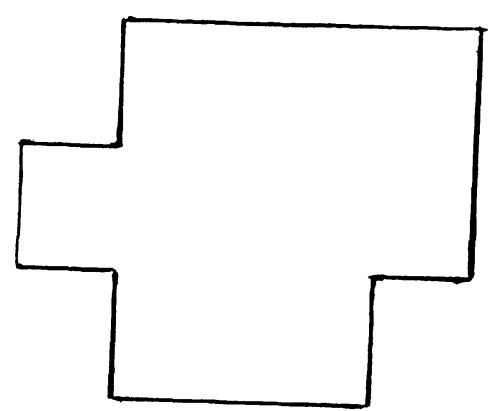

Figure 5. 
following $q_{2}, p_{4}$ near $q_{3}$ on the edge preceding $q_{3}$, and $p_{5}$ near $q_{3}$ on the edge following $\mathrm{q}_{3}$. (see Figure 4.) We have a contradiction, our supposition is false, and every two consecutive lnc points of $S$ must determine a segment parallel to one of the coordinate axes. Thus the assertion is established.

The rest of the argument is easy. Using the assertion above together with the fact that no three consecutive lnc points of $S$ are collinear, it is not hard to show that $S$ has at most 4 lnc points. Similarly, S cannot have exactly 3 Inc points, and since we are assuming that $n \geqslant 3$, we conclude that $n=4$. Hence by [8, Theorem 3], $S$ is a union of two closed convex sets.

Finally, using decomposition techniques from [8, Theorem 3], it is easy to show that $S$ may be expressed as a union of two rectangles, and the proof of Theorem 1 is complete.

To see that the number 5 in Theorem 1 is best possible, consider the following example.

EXAMPLE 2. Let $S$ be the set in Figure 5. Every 4 points of $S$ may be partitioned into sets $A$ and $B$ such that conv $A U \operatorname{conv} B \subseteq S$. However, $S$ is not a union of fewer than three convex sets.

In conclusion, it is interesting to observe that the result in Theorem 1 fails without the restriction on edges of $S$. In fact, there is no finite Helly number which characterizes unions of two convex sets, even for polygonal regions in the plane, and the Lawrence, Hare, Kenelly Theorem is best possible in this case. This is illustrated in Example 3.

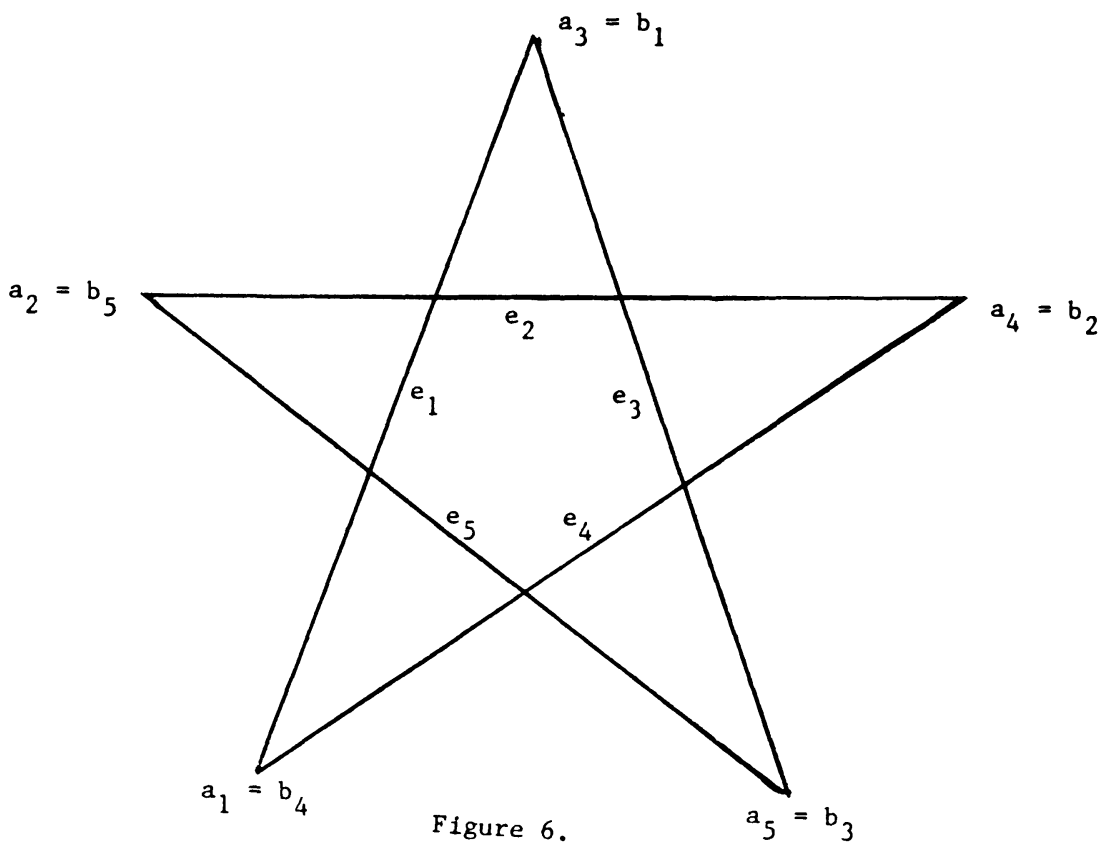

EXAMPLE 3. For $n \geqslant 2$, let $P(n)$ be a regular $(2 n+1)$-gon whose edges are labeled in a clockwise direction by $e_{1}, e_{2}, \ldots, e_{2 n+1}$. Adjusting our subscripts modulo $2 n+1$, for $1 \leqslant i \leqslant 2 n+1$, Let $s_{i} \equiv\left[a_{i}, b_{1}\right]$ be a segment containing $e_{i}$ such that $s_{i}$ meets $s_{i-2}$ at $a_{i}$ and $s_{i}$ meets $s_{1+2}$ at $b_{i}$. Finally, let 
$S(n)$ be the simply connected region determined by $U\left\{s_{i}: 1 \leqslant i \leqslant 2 n+1\right\}$. (The case for $n=2$ is illustrated in Figure 6.) Every $2 n$ points of $S(n)$ may be partitioned into sets $A$ and $B$ so that conv $A U$ conv $B \subseteq S$. However, the $2 n+1$ points $\left\{a_{i}: 1 \leqslant 1 \leqslant 2 n+1\right\}$ have no such partition, and $s(n)$ is not a union of fewer than three corvex sets.

\section{REFERENCES}

1. BREEN, M. A Krasnosel'skii-type theorem for unions of two starshaped sets in the plane, Pacific J. Math. (to appear).

2. DANZER, L. and GRÜNBAUM, B. Intersection properties of boxes in $R^{d}$, Combinatorica 2 (3) (1982), 237-246.

3. DANZER, L. GRÜNBAUM, B. and KLEE, V. Helly's theorem and its relatives, Proc. Symposia in Pure Math., Vol. VII (Convexity) (1963), 101-180.

4. LAWRENCE, J. F., HARE, W. R. Jr. and KENELLY, J. W. Finite unions of convex sets, Proc. Amer. Math. Soc. 34 (1972), 225-228.

5. LAY, S. R. Convex Sets and Their Application, John Wiley, New York, 1982.

6. TIETZE, H. Über Konvexheit im kleinen und im grossen und über gewisse den Pukten einer Menge zugeordnete Dimensionzahlen, Math. $\underline{\text { Z. }} 28$ (1928), 697-707.

7. TOSSAINT, G. T. and EL-GINDY, H. Traditional galleries are starshaped if every two paintings are visible from some common point, Amer. Math. Monthly (to appear).

8. VALENTINE, F. A. A three point convexity property, Pacific J. Math. 7 (1957), $1227-1235$.

9. VALENTINe, F. A. Convex Sets, McGraw HI11, New York, 1964. 


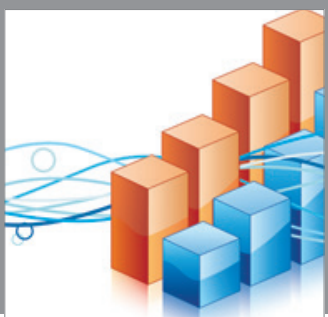

Advances in

Operations Research

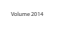

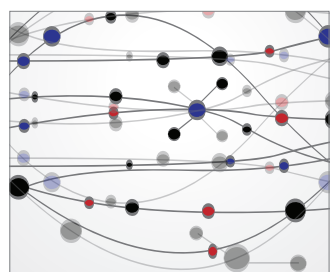

\section{The Scientific} World Journal
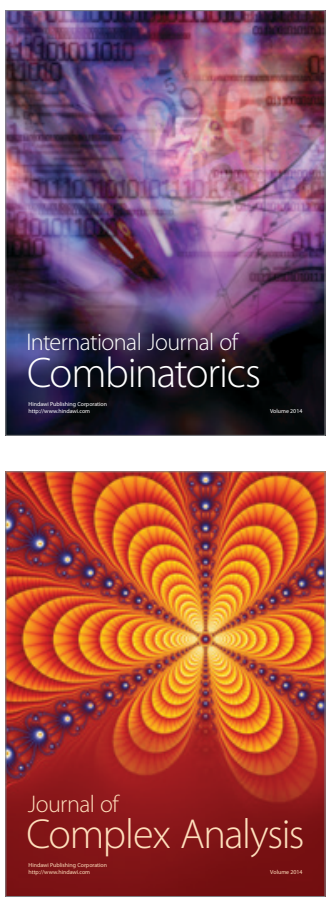

International Journal of

Mathematics and

Mathematical

Sciences
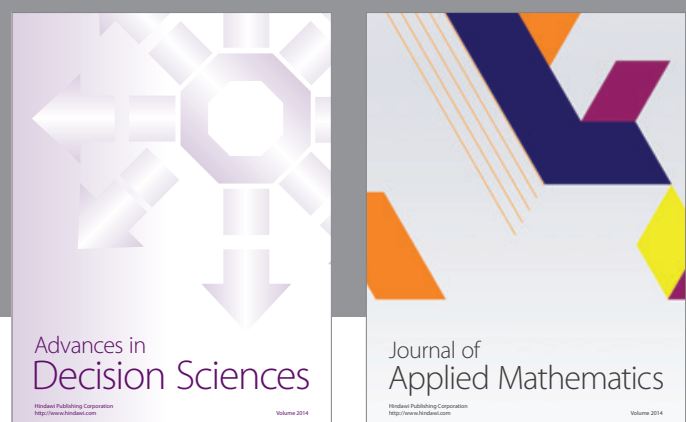

Journal of

Applied Mathematics
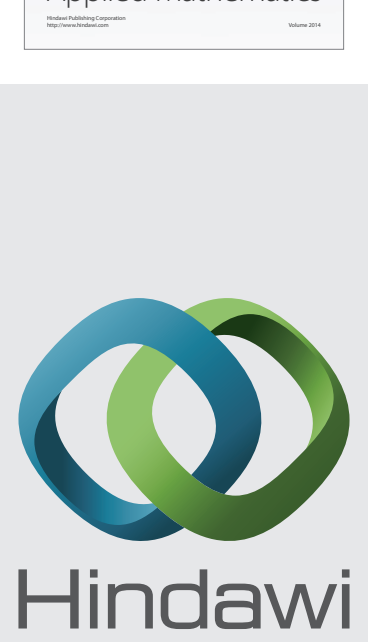

Submit your manuscripts at http://www.hindawi.com
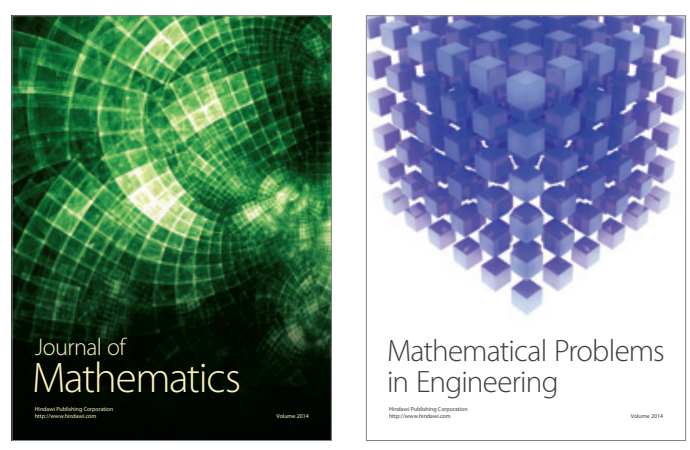

Mathematical Problems in Engineering
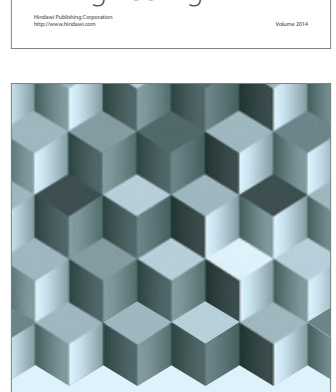

Journal of

Function Spaces
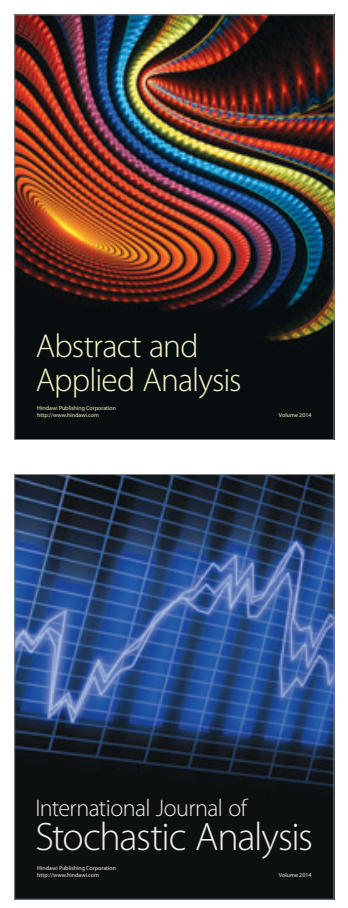

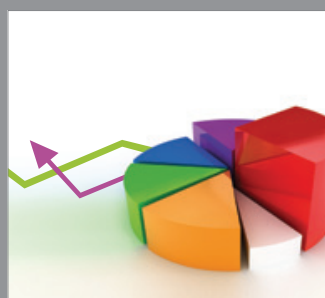

ournal of

Probability and Statistics

Promensencen
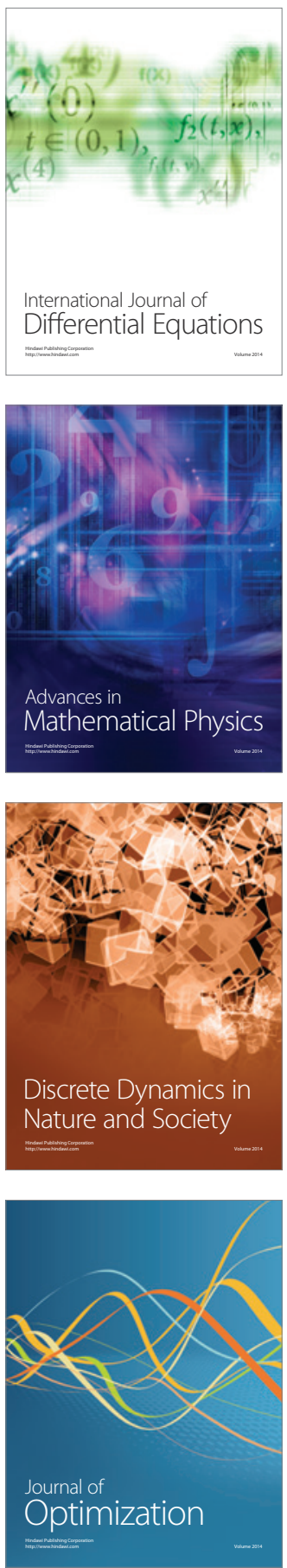